\title{
SÍNTESIS Y CARACTERIZACIÓN DE LA SENSIBILIDAD DE LAS NANOPARTÍCULAS DE ZnO MEDIANTE PRECIPITACIÓN HOMOGÉNEA IMPURIFICADAS CON Ni Y Cu
}

\author{
Veronica Estrella ${ }^{\mathrm{a}}$, Ma. Luz Olvera ${ }^{\mathrm{b}}$, I. Pedro Zaragoza ${ }^{\mathrm{a}}$, Arturo Maldonado ${ }^{\mathrm{b}}$, Benjamin $\operatorname{Vargas}^{\mathrm{a}}$ S. \\ Michelle Barrientos $^{\mathbf{a}}$
}

${ }^{a}$ Instituto Tecnológico de Tlalnepantla,Av. Tecnológico S/N Tlalnepantla Estado de México, México.

${ }^{b}$ Centro de Investigacion de Estudios Avanzados del Instituto Politécnico Nacional CINVESTAV-Zacatenco, La Laguna Ticoman, 07360 Ciudad de México, CDMX.

*vestrella@ittla.edu.mx

\section{Resumen}

La presente investigación se basa en la síntesis y caracterización de nanopartículas de óxido de zinc (ZnO) mediante una técnica de precipitación homogénea que, según los informes anteriores, tiene amplias expectativas de aplicación en sensores de gases en forma de película. El trabajo se centra en la síntesis de polvos y la caracterización de nanopartículas de $(\mathrm{ZnO})$, como la estructura cristalina y morfológica y las propiedades eléctricas al impurificarse con diversos metales (Ni) y $(\mathrm{Cu})$ en una atmósfera de propano $\left(\mathrm{C}_{3} \mathrm{H}_{8}\right)$. a diferentes temperaturas y concentraciones de gas. Las propiedades del sensor de polvos de $\mathrm{ZnO}$, impurificado con níquel $(\mathrm{Ni})$ en 4 inmersiones tienen un mayor grado de resistencia y sensibilidad que no contaminan, sensores de gas basados únicamente en $\mathrm{ZnO}$, por lo tanto películas de $\mathrm{ZnO}$ impurificadas con $\mathrm{Ni} \mathrm{y} \mathrm{Cu}$, en este trabajo se muestra que se obtuvieron valores de sensibilidad menores de 1 .

Palabras clave: precipitación homogénea, nanoparticulas, nanosensores.

\begin{abstract}
The present investigation is based on the synthesis and characterization of zinc oxide nanoparticles $(\mathrm{ZnO})$ by means of a homogeneous precipitation technique that, according to previous reports, has wide expectations of application in gas sensors in film form. The work focuses on the synthesis of powders and the characterization of $(\mathrm{ZnO})$ nanoparticles, such as the crystalline and morphological structure and the electrical properties when they are contaminated with various metals $(\mathrm{Ni})$ and $(\mathrm{Cu})$ in a propane atmosphere $(\mathrm{C} 3 \mathrm{H} 8)$. at different temperatures and gas concentrations. It was observed that the properties of the $\mathrm{ZnO}$ powder sensor, doped with nickel (Ni) in 4 dives, have a higher degree of resistance and sensitivity that do not pollute, gas sensors based solely on $\mathrm{ZnO}$, therefore $\mathrm{ZnO}$ films doped with $\mathrm{Ni}$ and $\mathrm{Cu}$, in this work it is shown that sensitivity values lower than 1 were obtained.
\end{abstract}

Keywords: homogeneous precipitation, nanoparticles, nanosensors.

\section{Introducción}

$\mathrm{El} \mathrm{ZnO}$ se encuentra en la naturaleza en forma de mineral como zincita [1], y comercialmente tiene una apariencia de polvo blanco. Éste es estable a temperatura ambiente y poco soluble en agua y es un óxido termo crómico, es decir, al calentar el óxido va cambiando de color. Se descompone a una temperatura de $1975^{\circ} \mathrm{C}$, generando vapor de $\mathrm{Zn} \mathrm{y} \mathrm{O}_{2}$ [2].

$\mathrm{El} \mathrm{ZnO}$ es un material semiconductor que se conoce como compuesto II-VI, por pertenecer sus componentes a los grupos IIB ( $\mathrm{Zn}$ ) y VIA (O). Tiene un peso molecular de 81.3694, el cual tiene un contenido de oxígeno de 50 \% (razón atómica), o bien, $19.66 \%$ (en masa) [3].
En años recientes el $\mathrm{ZnO}$ ha generado un gran interés debido a sus propiedades optoelectrónicas, considerándolo en la actualidad como un material que tiene numerosas aplicaciones en el campo de la optoelectrónica [4]. Entre sus aplicaciones se pueden destacar las siguientes: LED's, celdas solares y pantallas planas [5], transductores acústicos, varistores, sensores de gases, electrodos transparentes, ventanas ópticas en paneles solares, dispositivos emisores de campo, conductores transparentes [6], espejos térmicos [7], capas anti reflejantes y piezoeléctricos [4], entre otras. No obstante, el $\mathrm{ZnO}$ tiene también aplicación en otras áreas, como son: cosméticos, cremas de protección solar, tratamiento de acné, y productos bacteriostáticos, etc. [6]. 
La síntesis del $\mathrm{ZnO}$ en forma de película delgada o polvo ha sido llevada a cabo por diversas técnicas, tales como: descomposición térmica, método sol-gel, proceso hidrotérmico [8], spray pirolisis, depósito electroquímico, precipitación homogénea [9] y sputtering [10], entre otras.

El uso de sensores ha ido en aumento en las últimas décadas, teniendo como propósito la detección de humo, gases tóxicos, cocido de alimentos, humedad, entre otras aplicaciones. En nuestra actualidad podemos estudiar sensores de gases fabricados en forma de pastillas, películas delgadas, gruesas o en forma de polvos [9].

Los sensores más adecuados para la detección de gases reductores u oxidantes, son los sensores a base de óxidos metálicos, $\mathrm{OM}$, entre los que podemos mencionar al $\mathrm{Cr}_{2} \mathrm{O}_{3}, \mathrm{Cr}_{3} \mathrm{O}_{4}, \mathrm{NiO}, \mathrm{CuO}, \mathrm{MgO}, \mathrm{SrO}, \mathrm{BaO}, \mathrm{In}_{2} \mathrm{O}_{3}, \mathrm{WO}_{3}$, $\mathrm{TiO}_{3} \mathrm{~V}_{2} \mathrm{O}_{3}, \mathrm{Fe}_{2} \mathrm{O}_{3}, \mathrm{GeO}_{2}, \mathrm{Nb}_{2} \mathrm{O}_{5}, \mathrm{La}_{2} \mathrm{O}_{3}, \mathrm{SnO}, \mathrm{CeO}_{2}$ y $\mathrm{Nd}_{2} \mathrm{O}_{3}$. Sin embargo los más utilizados son el $\mathrm{ZnO}$ y el $\mathrm{SnO}_{2}$, debido a su costo bajo, menor toxicidad $\mathrm{y}$ disponibilidad [8].

Los OM tienen la característica de modificar su resistencia eléctrica superficial cuando la composición química de la atmosfera que los rodea es alterada.

Los óxidos semiconductores, como lo es en este caso el $\mathrm{ZnO}$, pueden ser utilizados para su aplicación en sensores de gases en diferentes presentaciones, en forma de polvos, o como en nuestro caso, como películas delgadas o gruesas. Podemos encontrar en la literatura reportes de sensores en base a $\mathrm{ZnO}$ [11- 14]. En este trabajo nuestra propuesta consiste en la preparación y caracterización de películas delgadas, fabricadas con base en nano partículas de $\mathrm{ZnO}$, para ser aplicadas a sensores de gas. El polvo se sintetizó por medio de la precipitación homogénea de una sal de zinc.

\section{Parte experimental}

Se realizó una mezcla del polvo obtenido de $\mathrm{ZnO}$ con $\mathrm{Cu}$ y $\mathrm{Ni}$ para mejorar su propiedades de conductividad eléctrica y en consecuencia la sensibilidad de tipo quimiresistivos son los dispositivos que se van a estudiar en este trabajo. Entre sus principales características se pueden mencionar las siguientes: procesos de fabricación simples, bajo costo de producción, tamaño compacto y una sencilla electrónica de medición. También presentan algunas desventajas, tales como: altas temperaturas de operación, una selectividad limitada y una sensibilidad modesta, sin embargo, estas propiedades pueden mejorarse con la adición de un catalizador adecuado al material sensor.

Los reactivos de partida y las cantidades empleadas fueron las siguientes: $1.5954 \mathrm{~g}$ de hidróxido de sodio ( $\mathrm{NaOH}$, Marca Sigma-Aldrich), en $100 \mathrm{ml}$ de metanol $\left(\mathrm{CH}_{4} \mathrm{O}\right)$; aparte se disolvió: $2.10 \mathrm{~g}$ de acetato de zinc di- hidratado $\left(\mathrm{Zn}\left(\mathrm{CH}_{3} \mathrm{COO}\right)_{2} \cdot 2 \mathrm{H}_{2} \mathrm{O}\right.$, Marca Alfa Aesa, $97 \%$ ) en $60 \mathrm{ml}$ de agua desionizada $\left(\mathrm{DI}-\mathrm{H}_{2} \mathrm{O}\right)$.

Se prepararon así 2 soluciones por separado, una con acetato de zinc (disuelto en agua desionizada), y la otra con hidróxido de sodio (disuelto en metanol).

\section{Resultados y discusión}

\subsection{Caracterización estructural de polvos de $\mathrm{ZnO}$}

Se muestra en la figura 1 la fase del compuesto, las propiedades cristalinas y el tamaño de cristal de las nanopartículas de $\mathrm{ZnO}$, se analizaron mediante difracción de rayos $\mathrm{X}$ (XRD) con un equipo marca PANalytical modelo XPERT-PRO, bajo las siguientes condiciones de operación: ánodo de $\mathrm{Cu}$ a un voltaje de $40.0 \mathrm{kv}$, corriente 20 MA una radiación $\mathrm{K} \alpha$ de $1.541874 \mathrm{~A}$ con una velocidad de escaneo de $0.039 \%$ s para 2 theta $(2 \theta)$ en un intervalo de 20 a $80^{\circ}$.

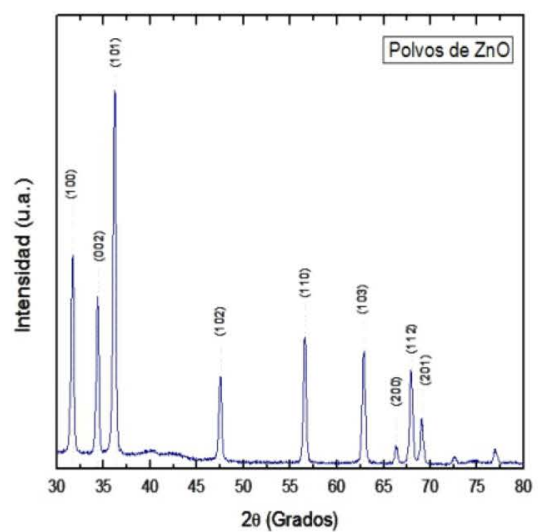

Fig. 1 Patrón de difracción de Rayos X para los polvos obtenidos por precipitación homogénea

Como se puede observar en las figuras 2, 3 de los resultados obtenidos, los sensores de gas en base sólo a $\mathrm{ZnO}$, muestran valores de sensibilidad bajos, en comparación con las películas de $\mathrm{ZnO}$ impurificadas con $\mathrm{Ni}$ y $\mathrm{Cu}$. Debido a que las películas de $\mathrm{ZnO}$ puro, muestran valores de sensibilidad menores a 1. Esto demuestra que, bajo las condiciones de operación, a 100, 200 y $300^{\circ} \mathrm{C}$, la quimisorción de gases sobre la superficie de $\mathrm{ZnO}$, no contribuye de manera significativa al incremento de portadores de carga dentro de la red de $\mathrm{ZnO}$. Cabe mencionar que el proceso de sensado de una película delgada semiconductora se basa en el hecho de 
que existe oxigeno quimisorbido sobre la superficie de dicha película el cual atrapa de uno a dos electrones de la red de $\mathrm{ZnO}$. Cuando un gas de tipo reductor entra en contacto sobre la superficie activada térmicamente, por cada molécula de oxigeno que desaloja, quedan uno o dos electrones libres en la red de la película, en este caso, $\mathrm{ZnO}$, los cuales ahora contribuyen al proceso de conducción del material. Este incremento en la concentración de portadores libres se refleja en las mediciones eléctricas, al encontrar una disminución significativa de la resistencia del material, $\mathrm{ZnO}$, como consecuencia de la liberación de un gran número de electrones, al desorberse el oxígeno molecular de la superficie del material.

Como se observa en las gráficas, la adición de Ni o $\mathrm{Cu}$ a la red de $\mathrm{ZnO}$, incrementa el valor de la Sensibilidad del sensor de gas. Como el resto de condiciones experimentales se mantienen fijas, es de esperarse que el incremento de la Sensibilidad de un sensor se deba a un mayor número de electrones liberados dentro de la red de $\mathrm{ZnO}$, promovido por la presencia de $\mathrm{Ni}$ o $\mathrm{Cu}$ en la superficie del sensor. Es decir, los iones metálicos de $\mathrm{Ni}$ o $\mathrm{Cu}$, localizados en la superficie del $\mathrm{ZnO}$, promueven una mayor desorción de las moléculas de oxigeno que se encuentra quimisorbido sobre la superficie de la película delgada.

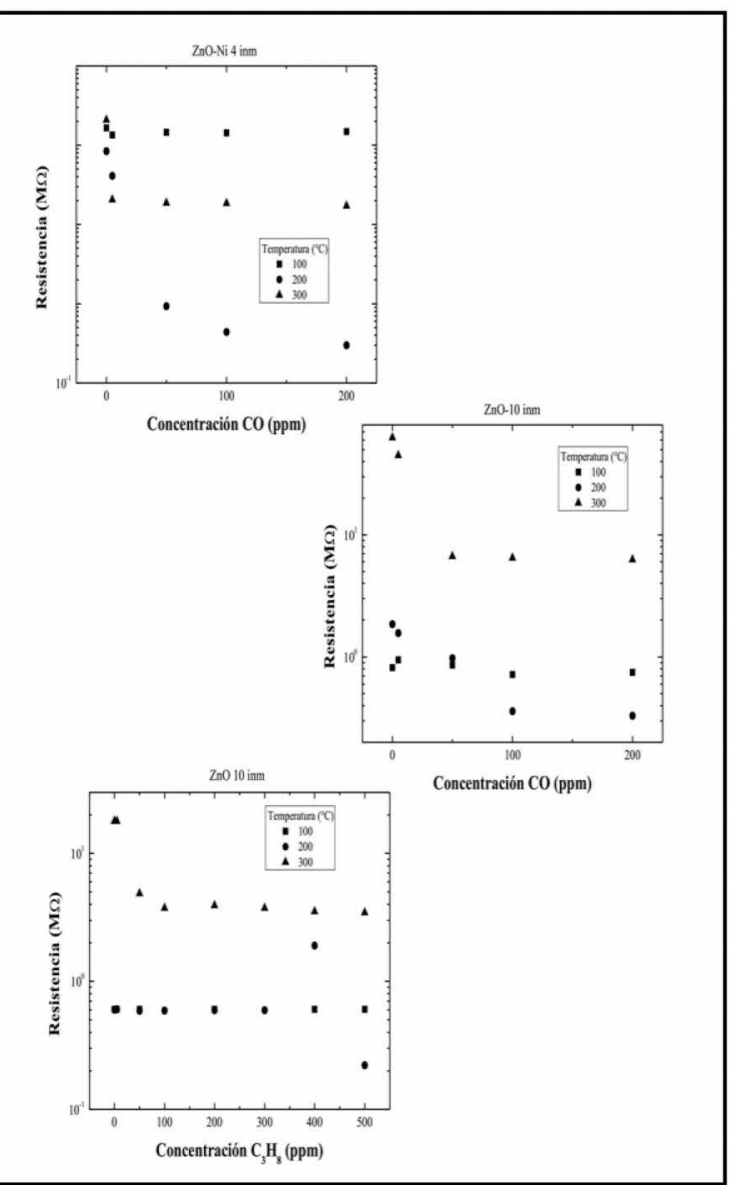

Fig. 2 Gráficas de la variación de la resistencia eléctrica de las películas de $\mathrm{ZnO}, \mathrm{ZnO}: \mathrm{Ni}$ y $\mathrm{ZnO}: \mathrm{Cu}$, en función de la concentración de propano, $\mathrm{C}_{3} \mathrm{H}_{8}$ y CO , a distintas temperaturas de operación,: 100,200 y $300^{\circ} \mathrm{C}$

La figura 3 muestra las gráficas de la variación de la resistencia eléctrica de las películas de $\mathrm{ZnO}, \mathrm{ZnO}$ : $\mathrm{Ni}$ y $\mathrm{ZnO}: \mathrm{Cu}$, en función de la concentración de propano, $\mathrm{C}_{3} \mathrm{H}_{8}$ y $\mathrm{CO}$, a distintas temperaturas de operación, para los sensores fabricados a base de polvos nanométricos de $\mathrm{ZnO}$. Estas mediciones se realizaron a diferentes concentraciones de $\mathrm{C}_{3} \mathrm{H}_{8}: 0,1,50,100,200,300,400$ y $500 \mathrm{ppm}, \mathrm{y}$ las temperaturas de operación del sensor fueron de: 100,200 y $300^{\circ} \mathrm{C}$.

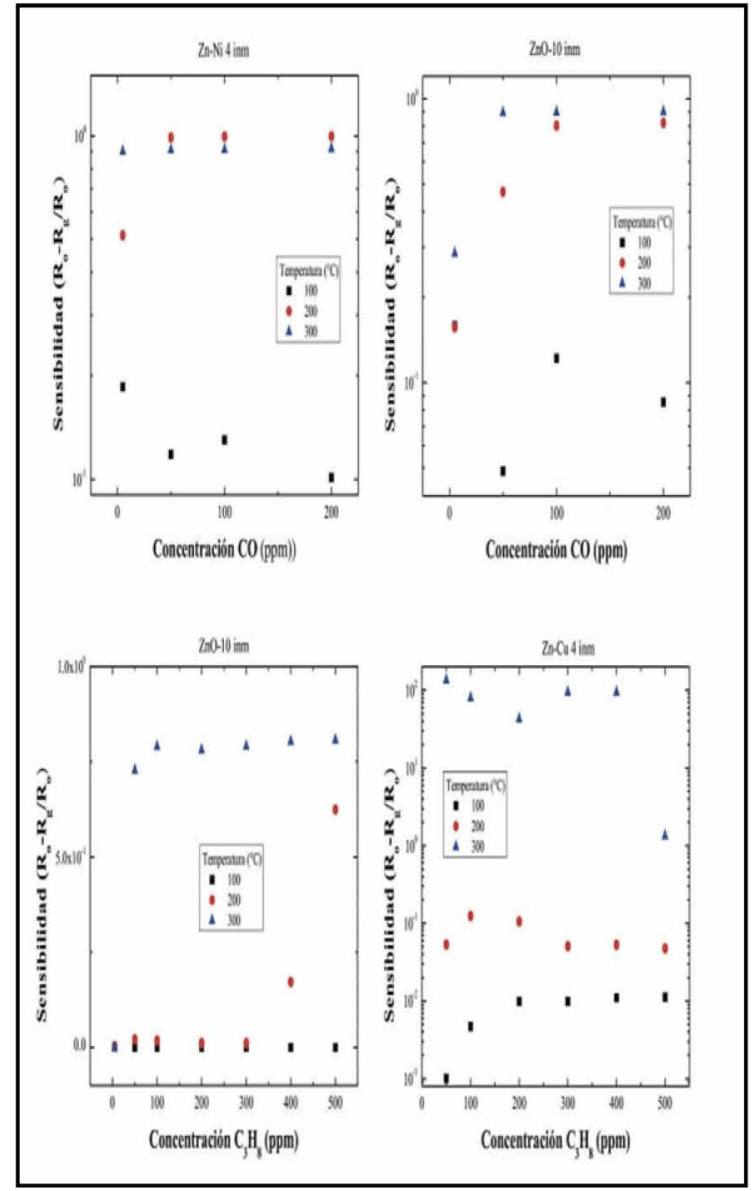

Fig. 3 gráficas de la variación de la resistencia eléctrica de las películas de $\mathrm{ZnO}, \mathrm{ZnO}: \mathrm{Ni}$ y $\mathrm{ZnO}: \mathrm{Cu}$, en función de la concentración de propano, $\mathrm{C}_{3} \mathrm{H}_{8}$ y CO, con temmperaturas de operación de: 100,200 y $300^{\circ} \mathrm{C}$.

\subsection{Películas de $\mathrm{ZnO}$}

Es importante mencionar que la resistencia eléctrica de referencia $(0 \mathrm{ppm})$ de las películas de $\mathrm{ZnO}$, sin impurificar, a bajas temperaturas de operación, es la misma, independientemente de la preparación, dentro del límite de medición de los aparatos. 
De los resultados obtenidos, y de manera general, podemos observar que a temperaturas de operación bajas, $100{ }^{\circ} \mathrm{C}$, no hay variación en la resistencia eléctrica de los sensores de película delgada de $\mathrm{ZnO}$. Tal es el caso de $\mathrm{ZnO}$ sin dopar, como se puede observar de la figura 3, que muestra un valor de resistencia constante, en todo el intervalo de concentración de gas, $\mathrm{CO}$ en este caso. Sin embargo, para temperaturas mayores, 200 y $300{ }^{\circ} \mathrm{C}$, se observa una variación en el valor de la resistencia de la película de $\mathrm{ZnO}$, a medida que se incrementa la concentración de gas $\mathrm{CO}$.

En el caso de gas Propano, se observó que a temperaturas de operación de 100 y $200{ }^{\circ} \mathrm{C}$ no hay un cambio significativo en la resistencia de las películas de $\mathrm{ZnO}$.

\subsubsection{Películas de ZnO: Ni}

En el caso de las películas de $\mathrm{ZnO}$ impurificadas con $\mathrm{Ni}$, empleadas para la detección de $\mathrm{CO}$, se observó nuevamente que a temperaturas bajas de operación del sensor, es decir, a $100 \mathrm{C}$, no hay variación significativa de la resistencia de la película. Al incrementar la temperatura de operación a 200 y 300 C, se observó una variación en la resistencia de la película, que muestra la interacción del gas con la superficie de dicha película.

\subsubsection{Películas de $\mathrm{ZnO}$ : $\mathrm{Cu}$}

En el caso de las películas de $\mathrm{ZnO}$ impurificadas con $\mathrm{Cu}$ se observó el mismo comportamiento a temperatura baja, es decir, a una temperatura de operación de $100 \mathrm{C}$, no varió la resistencia de la película con la concentración de los gases en la cámara. En cambio, al incrementar la temperatura de operación, de 200 a 300 C, se observó un cambio significativo en la resistencia de la película sensora. De hecho, este caso muestra el mejor desempeño como sensor de gas.

\subsection{Discusión}

La evaluación de los sensores de gases en base a su sensibilidad en función de dopante, concentración de gas y temperaturas de operación, se estudiaron en este trabajo. Como se puede observar de los resultados obtenidos, los sensores de gas en base sólo a $\mathrm{ZnO}$, muestran valores de sensibilidad bajos, en comparación con las películas de $\mathrm{ZnO}$ dopadas con $\mathrm{Ni}$ y $\mathrm{Cu}$. En efecto, las películas de $\mathrm{ZnO}$ puro, muestran valores de sensibilidad menores a 1 . Esto demuestra que, bajo las condiciones de operación, a saber, 100, 200 y $300 \mathrm{C}$, la quimisorción de gases sobre la superficie de $\mathrm{ZnO}$, no contribuye de manera significativa al incremento de portadores de carga dentro de la red de $\mathrm{ZnO}$. Hay que recordar que el proceso de sensado de una película delgada semiconductora se basa en el hecho de que existe oxigeno quimisorbido sobre la superficie de dicha película el cual atrapa de uno a dos electrones de la red de $\mathrm{ZnO}$. Cuando un gas de tipo reductor entra en contacto sobre la superficie activada térmicamente, por cada molécula de oxigeno que desaloja, quedan uno o dos electrones libres en la red de la película, en este caso, $\mathrm{ZnO}$, los cuales ahora contribuyen al proceso de conducción del material. Este incremento en la concentración de portadores libres se refleja en nuestro equipo de mediciones eléctricas, al encontrar una disminución significativa de la resistencia del material, $\mathrm{ZnO}$, como consecuencia de la liberación de un gran número de electrones, al de sorberse el oxígeno molecular de la superficie del material.

Como se observa de las Gráficas, la adición de Ni o $\mathrm{Cu}$ a la red de $\mathrm{ZnO}$, incrementa el valor de la Sensibilidad del sensor de gas. Como el resto de condiciones experimentales se mantienen fijas, es de esperarse que el incremento de la Sensibilidad de un sensor se deba a un mayor número de electrones liberados dentro de la red de $\mathrm{ZnO}$, promovido por la presencia de $\mathrm{Ni}$ o $\mathrm{Cu}$ en la superficie del sensor. Es decir, los iones metálicos de $\mathrm{Ni}$ o $\mathrm{Cu}$, localizados en la superficie del $\mathrm{ZnO}$, promueven una mayor desorción de las moléculas de oxigeno que se encuentra quimio absorbido sobre la superficie de la película delgada.

Los valores de sensibilidad, del orden de 100, hacen que el $\mathrm{ZnO}$ dopado con $\mathrm{Cu}$ sea el más adecuado sensor confiable de gas propano.

\section{Conclusiones}

De acuerdo a lo que se observa en las gráficas de las figuras 2 y 3 , la adición de $\mathrm{Ni}$ o $\mathrm{Cu}$ a la red de $\mathrm{ZnO}$, incrementa el valor de la sensibilidad del sensor de gas. Como el resto de condiciones experimentales se mantienen fijas, es de esperarse que el incremento de la sensibilidad de un sensor se deba a un mayor número de electrones liberados dentro de la red de $\mathrm{ZnO}$, promovido por la presencia de $\mathrm{Ni}$ o $\mathrm{Cu}$ en la superficie del sensor. Es decir, los iones metálicos de $\mathrm{Ni}$ o $\mathrm{Cu}$, localizados en la superficie del $\mathrm{ZnO}$, promueven una mayor desorción de las moléculas de oxigeno que se encuentra quimisorbido sobre la superficie de la película delgada.

\section{Referencias}

1. H. Odetti, E. Bottani (2006). Introducción a la química orgánica. Tercera edición.

2. V. E. Henrich, P.A. Cox, The surface sciencie of metal oxides, Cambridge University Press, Cambridge, 1994. 
3. J. A. McLeod, R. G. Wilks, N. A. Skorikov, L. D. Finkelstein, M. Abu-Samak, E. Z. Kurmaev, and A. Moewes.. Phys. Rev. B 81, (2010).

4. X. Liu, S. Cheng, H. Liu, S. Hu, D. Zhang and H. Ning, A survey on gas sensing technology, Sensors 12 (2012) 9635-9665.

5. A. Filipetti, V. Fiorentini, G. Capellini, A. Bosin,Phys. Rev. B 59 (1999) 8026.

6. http://www.uclm.es/profesorado/afantinolo/docencia /inorganica/Ficheros\%20pdf/ Tema\%207_IQ.pdf (accesado 18 de agosto del 2018)

7. http://www.zincoxidepowder.com (accesado el 15 de julio del 2018)

8. Samsonov, G. V. "The Oxide HandBook". Editorial IFI/PLENIUM. USA.1973.

9. Zhong Lin Wang. Journal of Physics: Condensed Matter. 2004. pp R829-R831.

10. Adachi, Sadao. "Handbook on Physical Properties of Semiconductors". Vol.3 Editor Kluwer Academic. 2004.

11. P. Cao, Y. Bai , D.X. Zhao , D.Z. Shen, Materials Science in Semiconductor Processing 14 (2011) 7377

12. T. Yingsamphancharoen, P. Nakarungsee, T.S. Herng, J. Ding , I.M. Tang, S. Thongmee, Journal of Magnetism and Magnetic Materials 419 (2016) 274-281

13. Xu Zi-qiang, Deng Hong, Li Yan, Cheng Hang, Materials Science in Semiconductor Processing 9 (2006) 132-135

14. D. Behera, B.S. Acharya, Journal of Luminescence 128 (2008) 1577-1586 